\title{
BMJ Open Metabolic syndrome and health-related behaviours associated with pre-oral cancerous lesions among adults aged 20-80 years in Yunlin County, Taiwan: a cross-sectional study
}

\author{
Chang-Cheng Chang, ${ }^{1}$ Ming-Shyan Lin, ${ }^{2}$ Yu-Tsung Chen, ${ }^{3,4}$ Liang-Tse Tu, ${ }^{5}$ \\ Su-Whi Jane, ${ }^{6}$ Mei-Yen Chen ${ }^{7}$
}

To cite: Chang $\mathrm{C}-\mathrm{C}$, Lin M-S, Chen Y-T, et al. Metabolic syndrome and health-related behaviours associated with pre-oral cancerous lesions among adults aged $20-80$ years in Yunlin County, Taiwan: a cross-sectional study. BMJ Open 2015;5: e008788. doi:10.1136/ bmjopen-2015-008788

- Prepublication history for this paper is available online. To view these files please visit the journal online (http://dx.doi.org/10.1136/ bmjopen-2015-008788).

C-CC, M-SL and Y-TC contributed equally.

Received 16 May 2015 Revised 19 November 2015 Accepted 23 November 2015

CrossMark

For numbered affiliations see end of article.

Correspondence to Dr Mei-Yen Chen; meiyen@gw.cgust.edu.tw

\section{ABSTRACT}

Objectives: To explore the associations of healthrelated behaviours, metabolic syndrome and risk factors in adults with pre-oral cancerous (POC) lesions in rural, disadvantaged communities with a high prevalence of oral cancer.

Design: A cross-sectional observational study. Setting: Community-based health survey in the western coastal area of Yunlin County, Taiwan.

Participants: 5161 adult residents participated in this study.

Outcome measures: Assessed parameters included oral leukoplakia, oral submucous fibrosis, fasting blood glucose, triglycerides, high-density lipoprotein cholesterol, blood pressure and waist circumference. Statistical analyses included descriptive statistics, $\chi^{2}$ tests and multivariate binary logistic regression.

Results: A high percentage of participants were found to have metabolic syndrome $(40 \%)$ and POC lesions $(7.3 \%)$. Participants with POC lesions tended to be male $(p<0.001)$, betel nut chewers $(p<0.001)$ and cigarette smokers $(p<0.001)$; have a low level of education $(p<0.001)$; seldom undergo dental checkups $(p<0.01)$; irregularly participate in physical activity $(p<0.01)$ and have metabolic syndrome $(p<0.01)$. Conclusions: Although male sex and disadvantaged socioeconomic status are non-modifiable factors associated with POC and metabolic syndrome in adults, several factors, notably health behaviours, are modifiable. Clinicians can reduce the incidence and consequences of POC by developing programmes for early detection, encouraging regular dental check-ups, and initiating individualised, health-promoting behaviour modification programmes for reducing risky behaviours associated with oral cancer.

\section{INTRODUCTION}

Oral cancer is the second most common cause of death from cancer in males in the southwestern coastal region of Taiwan. The

\section{Strengths and limitations of this study}

- Few studies have explored associations among health-related behaviours, metabolic syndrome and pre-oral cancerous lesions in disadvantaged areas.

- Pre-oral cancerous lesions are significantly associated with components of the metabolic syndrome.

- The outcome supports the value of a nurse-led community health-promotion programme for oral cancer prevention.

- Non-random sampling and individuals with erythroplakia and oral lichen planus were not included, which might limit the generalisability of the findings.

standardised mortality rate of oral cancer in this region $(34 / 100000)$ is higher than the national rate $(14.9 / 100000)$ and that throughout the rest of the world. ${ }^{12}$ Oral cancer is the fourth highest cause of cancer death of men aged 25-44 years in Taiwan; oral cancer mortality in this age group has increased $15 \%$ over the past 10 years. ${ }^{1}$ Each year, more than 2300 people die in Taiwan from oral cancer, and more than 5400 new cases are identified. ${ }^{1}$ Various oral mucosal changes can be transformed into pre-oral cancerous (POC) lesions, including leukoplakia, oral submucous fibrosis, lichenoid dysplasia, excipuliform and erythema. Undergoing oral mucous examination for identification of oral cancer can reduce oral cancer death by $40 \% .^{13}$

POC lesions can be readily detected by allowing non-medical personnel to visually inspect the oral cavity, a relatively easy aspect of routine examination. ${ }^{2}$ Important considerations could aid POC regression: with 
tobacco or betel nut use cessation, early detection and microsurgery can increase the 5 -year survival rate by $80 \%{ }^{12}$ Therefore, in epidemic areas of oral cancer, such as Yunlin County in Taiwan, early detection is an important and lifesaving strategy, as it may prevent progression to severe dysplasia and even carcinoma in situ and/or squamous cell carcinoma.

In the literature, the incidence of malignant transformation of oral submucous fibrosis ranges from $7 \%$ to $13 \% .{ }^{3}{ }^{4}$ Many studies indicate that oral leukoplakia, oral submucous fibrosis and oral erythroplakia are the most common oral mucosal diseases and have a very high malignant transformation rate..$^{5-7}$ Tilakaratne $e t a l^{3}$ indicated that the dense fibrosis and reduced vascularity of the corium of oral submucous fibrosis creates an environment that promotes the accumulation of carcinogens from betel nut chewing. The malignant transformation rate of oral leukoplakia has been found to range widely, from $13 \%$ to $19.8 \% .^{89}$

Alcohol, betel nut and cigarette (ABC) consumption has been reported to increase the incidence of oral cancer 123-fold compared to non-ABC consumption. ${ }^{10}$ Among the three components of $\mathrm{ABC}$ consumption, betel nut chewing, a widespread habit in southern Asian populations, is the most significant contributor to oral cancer development. ${ }^{11-13}$ Betel nut chewing is common in Taiwanese adults and is particularly high in industrial workers in India, ${ }^{13}$ especially those with low levels of education, as well as in rural minority populations. ${ }^{12}{ }^{13}$ Betel nut chewing has been associated with the development of oral leukoplakia, oral submucous fibrosis and oral cancer. ${ }^{14}$ The constituents of the betel nut, especially arecoline, are associated with the formation and progression of oral submucous fibrosis through interference with the deposition and/or degradation of extracellular matrix molecules such as collagen. The resulting nutritional deficiencies may induce a synergistic effect by contributing to epithelial atrophy. ${ }^{7}$ When combined with betel nut chewing, cigarette smoking significantly contributes $(86.5 \%)$ to the risk of oral leukoplakia and oral submucous fibrosis. ${ }^{14} 15$

In a community-based integrated screening programme conducted between 2003 and 2008, Yen et $a l^{16}$ examined 79940 participants aged 20 years or older undergoing oral mucous examinations as well as health check-ups for metabolic syndrome in Keelung, Taiwan. They found that metabolic syndrome in betel nut chewers was significantly associated with oral premalignancy after adjusting for the confounding factors of age, sex, betel nut chewing, smoking and alcohol drinking, and that this association persisted even in nonchewers when other risk factors were controlled. Recently, several studies reported that metabolic syndrome is associated with many types of cancers, such as liver cancer, oesophageal cancer, colon cancer and oral cancer. ${ }^{17}{ }^{18}$ Investigations conducted in Germany ${ }^{19}$ and India ${ }^{13}$ found that occupation and educational inequality are significantly associated with oral cancer.
Our research team (Guo et $\left.a l^{20}\right)$ had previously reported that betel nut chewing and cigarette smoking are negatively associated with health-promoting behaviours after adjustment for socioeconomic factors. Further delineation of these relationships would be enhanced by exploration of the relationship among health-related behaviours, metabolic syndrome and POC lesions in Taiwan.

Despite numerous studies reporting that $\mathrm{ABC}$ consumption is associated with the development of oral and laryngeal cancers, the associations among $\mathrm{ABC}$ consumption, health-promoting behaviours, metabolic syndrome and POC lesions are rarely discussed in primary healthcare. Nevertheless, ABC consumption is well known as a mechanism underlying the development of oral cancer, ${ }^{14} 21-23$ and several health programmes for ABC cessation have been available in Taiwan for many years. ${ }^{1}$ Despite this knowledge and the availability of these programmes, few studies have explored the associations among health-related behaviours, metabolic syndrome and risk factors in adults with POC in communities with a high prevalence of oral cancer in disadvantaged areas. Therefore, the study objective was to determine the associations of metabolic syndrome, health-related behaviours and POC lesions.

\section{METHODS}

\section{Design, sample and setting}

This study was a series report of a nurse-led longitudinal study of health promotion for community health development in the southwestern coastal area of Yunlin County, Taiwan. Using a cross-sectional descriptive design, a community-based health screening survey of adult residents was conducted between August 2011 and July 2012. The inclusion criteria were (1) aged from 20 to 80 years, (2) full independence in performing activities of daily living, (3) ability to complete the questionnaires in Mandarin or in a Taiwanese dialect either by selfadministration or during an interview and (4) ability to understand and sign an informed consent form before enrolment in the study. Exclusion criteria were (1) a serious learning difficulty or (2) an inability to answer questionnaires. The participants were selected using convenience samples from 27 villages of the southwestern coastal area. A total of 6823 community adults participated in the health survey. In total, 5161 participants met the criteria and enrolled for the full statistical analysis.

\section{Procedure and ethical considerations}

This study was conducted in collaboration with a local hospital through a community health-screening programme and was approved by the institutional review board ethical committee. After research assistants had explained the study objectives to the potential participants, those who agreed to participate signed an informed consent form. 
In order to avoid measurement error, 12 research assistants were separated into six pairs before the start of the project and were asked to interview an elder in the community activity centre, which allowed us to confirm a $90 \%$ correct response rate of inter-rater reliability among these six pairs. Face and content validity of the instrument were judged to be good (CVI=0.90-0.92) by a panel of five experts-a faculty member in public health and health education, endocrine and metabolic physicians, and nursing faculty members teaching health promotion. Some items within the instruments were revised according to experts' suggestions. All research assistants were senior nursing students and were trained for $4 \mathrm{~h}$ by the investigators in two consequent training programmes. In session 1, we focused on practicing interview skills. In session 2, all research assistants were grouped into six pairs to pretest and become familiar with all items of the questionnaire. To reduce the potentially negative influence of inter-rater reliability, consistency among participating dentists was ensured in three ways: (1) all were well trained with $>5$ years of clinical experience, (2) they tested for and discussed POC using clinical photos before the examination and (3) the consensus for evaluating uncertain oral lesions was continually adjusted during the health screening programme.

\section{Measurements}

1. Participant characteristics. Data regarding participant characteristics were collected by instructing the participants to answer items regarding their age (year of birth), sex, self-perceived economic status (How do you feel about your economic situation? Good, fair/ not bad, poor/bad) and educational attainment (highest educational year completed or school level at which the participant graduated).

2. Identification and examination of POC lesion. Examination of the oral mucosa was performed by four experienced dentists, assisted by nurses, according to the WHO criteria. ${ }^{24}$ All participants received a conventional clinical check-up and an additional examination for POC lesions by one experienced dentist under an incandescent overhead light. In the first step, the participant was seated in a regular chair, and any intraoral prostheses (dentures or partial dentures) were removed before starting the examination. The extraoral and perioral tissues were examined first, followed by the intraoral tissues. Major POC lesions such as oral leukoplakia and oral submucous fibrosis were identified via mucosal screening. The second step was checking for uncertain oral mucosa lesions by a second examiner, and a final diagnosis was reached by agreement between both dentists. For some special cases such as thick leukoplakia, submucous fibrosis and verrucous hyperplasia, the examiner strongly recommended further biopsy after the health screening.

3. Measurement of metabolic syndrome. Metabolic syndrome was defined according to the Health Promotion
Administration ${ }^{1}$ nationwide standard as the presence of three or more of the following abnormal biomarkers: (i) fasting blood glucose level $>100 \mathrm{mg} / \mathrm{dL}$, (ii) triglyceride level $>150 \mathrm{mg} / \mathrm{dL}$, (iii) high-density lipoprotein (HDL) cholesterol level $<40 \mathrm{mg} / \mathrm{dL}$ for males or $50 \mathrm{mg} / \mathrm{dL}$ for females, (iv) blood pressure $>130 / 85 \mathrm{~mm} \mathrm{Hg}$ and (v) waist circumference (WC) $>90 \mathrm{~cm}$ for males and $80 \mathrm{~cm}$ for females. Venous blood samples were drawn after an $8 \mathrm{~h}$ fast, by the collaborating hospital. Blood pressure and WC were measured at the time of the study using standard procedures. WC was measured between the last rib margin and the iliac crest (ie, the mid-abdominal distance) and was used as an indication of central obesity. ${ }^{1}$

4. Health-related behaviours. The following health-related behaviours were assessed: (i) ABC consumption: participants were classified as either (a) never having engaged in $\mathrm{ABC}$ consumption or (b) currently, or having ceased $\mathrm{ABC}$ consumption, if they were currently engaging in or had ceased $\mathrm{ABC}$ consumption, respectively; (ii) physical activity level: participants were classified as either (a) engaging in regular physical activity if they usually exercised for $>30 \mathrm{~min} /$ day three times/week or for $150 \mathrm{~min} /$ week or (b) irregularly engaging in physical activity if they never or only sometimes engaged in physical activity; (iii) dental check-ups: participants were classified as either (a) regularly participating in dental check-ups if they visited a dental clinic for a check-up at least once per year or (b) seldom participating in dental check-ups if they had never participated in dental check-ups or irregularly participated in dental check-ups (ie, less than once per year).

\section{Statistical analysis}

The data regarding demographic characteristics, healthrelated behaviours and metabolic syndrome components were dichotomised for comparison using the $\chi^{2}$ test between the group of participants with POC lesions, and the participants without POC lesions. The relationships among these factors and POC lesions were investigated using multivariate binary logistic regression. The results were presented as the adjusted OR with the corresponding 95\% CI. All data analyses were conducted using SPSS software V.20 (SPSS Inc, IBM Corp, Armonk, New York).

\section{RESULTS}

Participants had a mean age of 49.1 years ( $\mathrm{SD}=16.0$ years), $55.8 \%$ were female and $377(7.3 \%)$ were identified as having POC lesions. Regarding the subtype of POC lesion, $279(74 \%)$ had oral leukoplakia and $98(26 \%)$ had oral submucous fibrosis. Participants with POC lesions were significantly $(\mathrm{p}<0.001)$ more likely to be men, less educated, of poor socioeconomic status, alcohol drinkers, betel nut chewers and cigarette smokers; to seldom participate in dental check-ups; and 
to irregularly engage in physical activity, compared to participants without POC lesions. In contrast, no significant difference in age was found between the participants with and without POC lesions (table 1).

Regarding the components of metabolic syndrome, participants with POC lesions were significantly $(p<0.001)$ more likely to have high blood pressure, high fasting blood glucose levels, high triglyceride levels, low HDL cholesterol levels and a high WC compared to participants without POC lesions. According to analysis of these components, 2062 (40\%) participants had metabolic syndrome, a significantly higher percentage of whom had POC lesions compared to participants without metabolic syndrome ( $10 \%$ vs $5 \%, \mathrm{p}<0.001$; table 2$)$.

Variables found to be significantly associated with POC lesions and the presence of metabolic syndrome were introduced into the multivariate logistic regression model. The results indicated that the covariates independently associated with a higher risk of POC lesions were male sex $(\mathrm{OR}=7.24, \mathrm{p}<0.001)$, low level of education $(\mathrm{OR}=2.05, \mathrm{p}<0.001)$, betel nut chewing $(\mathrm{OR}=2.98$, $\mathrm{p}<0.001)$, cigarette smoking $(\mathrm{OR}=1.95, \mathrm{p}<0.001)$, seldom participating in dental check-ups $(\mathrm{OR}=1.42, \mathrm{p}<0.01)$, irregularly engaging in physical activity $(\mathrm{OR}=1.41$, $\mathrm{p}<0.01)$ and having metabolic syndrome $(\mathrm{OR}=1.39$, $\mathrm{p}<0.01$; table 3 ).

\section{DISCUSSION}

Although random sampling was not performed, the analysis of a large, rural sample yielded findings that were sufficiently reliable to assist in the development of nursing intervention programmes for primary healthcare and promotion in oral cancer prevention. Among the findings regarding the association between POC lesions and risk factors, two were especially illuminating: (1) except for male sex and poor socioeconomic status, several factors were modifiable, including betel nut chewing, cigarette smoking, seldom participating in regular dental check-ups and irregularly engaging in exercise and (2) the presence of a strong association between metabolic syndrome and POC lesions.

The study findings agree with those of several previous studies in Taiwan and India. Among them, Guo et $a l^{20}$ and Arora $e t \mathrm{al}^{13}$ identified that betel nut chewing and cigarette smoking were negatively associated with health behaviours, such as poor oral hygiene and low levels of exercise, and that demographic factors such as male sex, low education level and low socioeconomic level were associated with POC lesions. Therefore, early prevention of betel nut chewing and cigarette smoking, through participation in individualised and culturally appropriate programmes, may lower the incidence and mortality rate from oral cancer. Among potential strategies to consider

Table 1 Demographic characteristics and health-related behaviours among rural residents with POC lesions $(n=5161)$

\begin{tabular}{|c|c|c|c|}
\hline Variable & $\begin{array}{l}\text { Non-POC } \\
(n=4784)\end{array}$ & $\begin{array}{l}\text { POC } \\
(n=377)\end{array}$ & p Value \\
\hline Age (years) & & & 0.637 \\
\hline$<65$ & $3822(93 \%)$ & $305(7 \%)$ & \\
\hline$\geq 65$ & 962 (93\%) & $72(7 \%)$ & \\
\hline Gender & & & $<0.001$ \\
\hline Female & $2842(99 \%)$ & $38(1 \%)$ & \\
\hline Male & $1942(85 \%)$ & $339(15 \%)$ & \\
\hline Education level & & & $<0.001$ \\
\hline High school and above & $2122(95 \%)$ & $108(5 \%)$ & \\
\hline Elementary school and below & $2662(91 \%)$ & $269(9 \%)$ & \\
\hline Self-perceived economic status & & & $<0.001$ \\
\hline Feel good or fair & $3848(93 \%)$ & $275(7 \%)$ & \\
\hline Feel poor or bad & $936(90 \%)$ & $102(10 \%)$ & \\
\hline Alcohol use & & & $<0.001$ \\
\hline Never & $3669(95 \%)$ & $180(5 \%)$ & \\
\hline Current and cessation & $1115(85 \%)$ & $197(15 \%)$ & \\
\hline Betel chewing & & & $<0.001$ \\
\hline Never & $4411(95 \%)$ & $209(5 \%)$ & \\
\hline Current and cessation & $373(69 \%)$ & $168(31 \%)$ & \\
\hline Cigarette smoking & & & $<0.001$ \\
\hline Never & $4069(96 \%)$ & $169(4 \%)$ & \\
\hline Current and cessation & 715 (77\%) & $208(23 \%)$ & \\
\hline Dental check-ups & & & $<0.001$ \\
\hline Regular (>1/year) & $2182(95 \%)$ & $110(5 \%)$ & \\
\hline Seldom/never & 2602 (91\%) & 267 (9\%) & \\
\hline Physical activity & & & $<0.001$ \\
\hline Regular* & $2411(94 \%)$ & $145(6 \%)$ & \\
\hline Irregular & 2373 (91\%) & $232(9 \%)$ & \\
\hline
\end{tabular}


Table 2 Association of metabolic syndrome and its components with POC lesions

\begin{tabular}{|c|c|c|c|}
\hline Variable & $\begin{array}{l}\text { Non-POC } \\
(n=4784)\end{array}$ & $\begin{array}{l}\text { POC } \\
(n=377)\end{array}$ & p Value \\
\hline BMI (body mass index) kg/m² & & & $<0.001$ \\
\hline Normal/underweight & $2273(95 \%)$ & $119(5 \%)$ & \\
\hline Overweight/obesity & $2511(91 \%)$ & $258(9 \%)$ & \\
\hline Systolic blood pressure (mm Hg) & & & $<0.001$ \\
\hline$\leq 129$ & $2155(94 \%)$ & $127(6 \%)$ & \\
\hline$\geq 130$ & 2629 (91\%) & $250(9 \%)$ & \\
\hline Diastolic blood pressure $(\mathrm{mm} \mathrm{Hg})$ & & & $<0.001$ \\
\hline$\leq 84$ & $2830(94 \%)$ & $170(6 \%)$ & \\
\hline$\geq 85$ & $1954(90 \%)$ & $207(10 \%)$ & \\
\hline Fasting blood glucose (mg/dL) & & & $<0.001$ \\
\hline$\leq 100$ & $3679(94 \%)$ & $250(6 \%)$ & \\
\hline$\geq 101$ & $1105(90 \%)$ & $127(10 \%)$ & \\
\hline Triglyceride (mg/dL) & & & $<0.001$ \\
\hline$\leq 149$ & 3989 (94\%) & $240(6 \%)$ & \\
\hline$\geq 150$ & 795 (85\%) & $137(15 \%)$ & \\
\hline $\mathrm{HDL}(\mathrm{mg} / \mathrm{dL})$ & & & $<0.001$ \\
\hline Male $\geq 40 /$ female $\geq 50$ & 3814 (94\%) & $263(6 \%)$ & \\
\hline Male $<40 /$ female $<50$ & $970(89 \%)$ & $114(11 \%)$ & \\
\hline Waist circumference (cm) & & & $<0.001$ \\
\hline Male $\leq 89 /$ female $\leq 79$ & 2608 (94\%) & $172(6 \%)$ & \\
\hline Male $\geq 90 /$ female $\geq 80$ & $2176(91 \%)$ & 205 (9\%) & \\
\hline Metabolic syndrome* & & & $<0.001$ \\
\hline No & 2933 (95\%) & $166(5 \%)$ & \\
\hline Yes & $1851(90 \%)$ & $211(10 \%)$ & \\
\hline
\end{tabular}

${ }^{*}$ Metabolic syndrome $\geq$ three risk factors (eg, abnormal blood pressure, fasting blood glucose, triglyceride, HDL, waist circumference). $\mathrm{BMI}$, body mass index; HDL, high-density lipoprotein; POC, pre-oral cancer.

are increasing health literacy, using adequate media and providing health-promotion programmes for oral cancer prevention targeted towards men. The study findings can be used to stimulate further research exploring methods of preventing initiation of risky health behaviours; promoting healthy behaviours; and assisting in cessation of alcohol drinking, betel nut chewing and cigarette smoking.

As shown in table 1, the percentage of ABC consumption and POC lesions were $15 \%, 31 \%$ and $23 \%$, respectively. Surprisingly, after adjusting for confounders,

Table 3 Logistic regression of factors associated with pre-oral cancer lesions

\begin{tabular}{llr}
\hline Predictor & aOR (95\% Cl of OR) & p Value \\
\hline Male gender & $7.24(5.01$ to 10.47$)$ & $<0.001$ \\
Low education level & $2.05(1.57$ to 2.67$)$ & $<0.001$ \\
Betel chewing & $2.98(2.25$ to 3.94$)$ & $<0.001$ \\
Cigarette smoking & $1.95(1.47$ to 2.59$)$ & $<0.001$ \\
$\begin{array}{l}\text { Seldom having dental } \\
\text { check-ups }\end{array}$ & $1.42(1.10$ to 1.84$)$ & 0.007 \\
$\begin{array}{l}\text { Irregular physical } \\
\text { activity }\end{array}$ & $1.41(1.11$ to 1.79$)$ & 0.005 \\
$\begin{array}{l}\text { Presence of metabolic } \\
\text { syndrome }\end{array}$ & $1.39(1.10$ to 1.77$)$ & 0.006 \\
\hline aOR, adjusted OR. & & \\
\end{tabular}

alcohol consumption was not found to be associated with POC lesions. This finding agrees with several studies, such as that by Chung et $a l^{25}$ which found that 79 participants whose risky behaviours were limited to alcohol drinking did not develop oral submucous fibrosis. Lee $e t a l^{6}$ also suggested that alcohol consumption is not a risk factor for POC lesions. Interestingly, Guo et $a t^{20}$ found that alcohol users tend to have higher education levels, higher socioeconomic status, perform more physical activity and undergo regular dental checkups, all of which were associated with a lower incidence of POC lesions. However, this association should be viewed with caution, as the study did not measure the consumption level of alcohol. Further studies should take the type and amount of alcohol into account.

Twenty years ago, the Taiwanese government launched a national health insurance programme that currently covers more than $99 \%$ of population. Unfortunately, $56 \%(n=2869)$ of the current participants reported that they seldom or never undergo regular dental check-ups. A possible reason for this is a lack of resources to devote to healthcare, making it difficult to travel to a dental clinic located outside of a rural area. It is thus urgent to initiate a special dental programme that conducts annual POC screening and teaches self-management of oral hygiene for less-educated men who are ABC users. Fortunately, the Taiwanese government has, over the last 
10 years, implemented several strategies to reduce the prevalence of $\mathrm{ABC}$ consumption, including legislation to increase the price of or tax on ABC. ${ }^{1}$

Despite these measures, a high incidence of $\mathrm{ABC}$ consumption persists among adults residing along the western coast who have been diagnosed with oral cancer. One reason for this persistence may be a lack of comprehension regarding the association between $\mathrm{ABC}$ consumption and the aetiology and mechanism underlying POC lesion development. Another reason may be a lack of appropriate understanding regarding the low adherence to health and education policies in rural areas, particularly among socioeconomically disadvantaged community residents. Thus, we suggest a low level of health literacy, defined as a limited ability to obtain, process and understand basic health information and the services needed to make appropriate health decisions, as a potential reason for continued $\mathrm{ABC}$ use and consequent POC susceptibility in underserved rural areas.

Consistent with several previous studies, ${ }^{17}{ }^{18}$ POC lesions were found to be significantly associated with the components of metabolic syndrome in the present study, including central obesity, high blood pressure, hyperglycaemia and dyslipidaemia. Tsai et $a l^{23}$ found that betel nut chewing, cigarette smoking, hypertension and dyslipidaemia interact in a manner that increases coronary artery disease risk. The $40 \%$ prevalence of metabolic syndrome found in this study is much higher than the nationwide prevalence of $20 \%$ of adults in Taiwan ${ }^{26}$ and $25 \%$ of adults in the $\mathrm{USA},{ }^{27}$ although the prevalence is rapidly rising throughout the world.

Karmali et $a l^{27}$ recently reported the possible mechanisms underlying metabolic syndrome and its relationship to cancer development may be the mitogenic effects of obesity and insulin resistance, which alter lipid signalling, and affect adipokine and inflammatory cytokine levels. Although the exact pathway linking metabolic syndrome and oral cancer is unknown, reducing the incidence of metabolic syndrome and inflammatory-induced diseases through health-promotion programmes appears to be an important goal to reduce the incidence of oral cancer. Therefore, we are initiating health-promotion programmes to promote health literacy ${ }^{28}$ among disadvantaged people living in areas with a high prevalence of oral cancer. The health-promotion programmes will provide culturally oriented educational materials for men, as well as specific language-sensitive pamphlets adapted to emphasise the role of $\mathrm{ABC}$ use, and the importance of physical activity and dental check-ups for POC prevention.

\section{Study limitations and future directions}

This study has several limitations. First, the use of crosssectional data precludes the inference of causal relationships. Second, the use of self-reported data might lead to underestimation of health-related behaviours such as the frequency or amount of alcohol consumption, betel nut chewing and cigarette smoking. Third, selection and recall bias need to be considered because the participants might have been incapacitated by various health conditions. Fourth, because of the use of non-random sampling, and because individuals with erythroplakia and oral lichen planus were not included, the generalisability of the findings may be limited. Fifth, the $\kappa$ statistic was not used to test inter-rater reliability of dentists in this study, although we did adopt multiple steps to ensure reliability and consistency. Further research should validate the POC portion of the dental examination. Future research should also explore methods of preventing initiation of risky health behaviours; promoting healthy behaviours; and assisting with cessation of alcohol drinking, betel nut chewing and cigarette smoking. Primary healthcare providers should conduct health-promotion programmes related to the early detection of oral health and physical activity through community-based health development, specifically for socioeconomic minorities and males in rural areas.

\section{CONCLUSION}

Despite some limitations, this study demonstrated associations among health-related behaviours, metabolic syndrome and POC lesions, in disadvantaged areas. In addition, POC lesions were significantly associated with the components of metabolic syndrome. The outcome supports the value of a nurse-led community healthpromotion programme for oral cancer prevention. In rural communities in southwestern Taiwan where oral cancer prevalence is high, participants with POC lesions were likely to have poor health-related behaviours, such as betel nut chewing, cigarette smoking, irregular engagement in exercise and infrequent undergoing of dental check-ups. POC lesions were significantly associated with the components of metabolic syndrome, including central obesity, high blood pressure, high fasting blood glucose and triglyceride levels and low HDL cholesterol levels.

To reduce the consequences of POC lesions in rural areas, clinicians and primary healthcare providers need to understand the health behaviours and risk factors specific to a particular population, and to lead community health promotion efforts through development of efficient strategies, including specific, individualised, culturally tailored health-promotion programmes that promote health literacy.

\section{Author affiliations}

${ }^{1}$ Division of Plastic Surgery, Head and Neck Cancer Team, Chang Gung Memorial Hospital, Chiayi, Taiwan

${ }^{2}$ Division of Cardiology, Chang Gung Memorial Hospital, Yunlin, Taiwan ${ }^{3}$ Department of Dermatology, Shuang Ho Hospital, Taipei Medical University, Taipei, Taiwan

${ }^{4}$ School of Public Health, College of Public Health and Nutrition, Taipei Medical University, Taipei, Taiwan

${ }^{5}$ Division of Dentistry, Cancer Center, Chang Gung Memorial Hospital, Chiayi, Taiwan

${ }^{6}$ Nursing Department, Chang Gung University of Science and Technology, Taoyuan, Taiwan 
${ }^{7}$ Graduate Institute of Nursing, Chang Gung University of Science and Technology, Chiayi, Taiwan

Acknowledgements The authors would like to acknowledge, the paper was edited by a native English speaker, Mr David K of Editage Company, Taiwan.

Contributors C-CC, Y-TC and M-SL gave valuable contributions to the design and manuscript of the present study. M-YC was the principal author and responsible for the study. L-TT and S-WJ jointly drafted the manuscript.

Funding This work was supported by grants from the Taiwan Formosa Plastic Company (FCRPF690011) and Chang Gung Memorial Hospital (BMRP148)

Competing interests None declared.

Ethics approval Chang Gung Memorial Hospital.

Provenance and peer review Not commissioned; externally peer reviewed.

Data sharing statement No additional data are available.

Open Access This is an Open Access article distributed in accordance with the Creative Commons Attribution Non Commercial (CC BY-NC 4.0) license, which permits others to distribute, remix, adapt, build upon this work noncommercially, and license their derivative works on different terms, provided the original work is properly cited and the use is non-commercial. See: http:// creativecommons.org/licenses/by-nc/4.0/

\section{REFERENCES}

1. Health Promotion Administration, Ministry of Health and Welfare. Oral cancer. http://www.hpa.gov.tw/BHPNet/Web/HealthTopic/Topic. aspx?id=201109140001 (accessed 8 Apr 2015).

2. World Health Organization, WHO. Screening for oral cancer. http:// www.who.int/oral_health/events/Global_consultation/en/index.html (accessed 20 Feb 2015).

3. Tilakaratne WM, Klinikowski MF, Saku T, et al. Oral submucous fibrosis: review on aetiology and pathogenesis. Oral Oncol 2006; $42: 561-8$

4. Murti PR, Bhonsle RB, Pindborg JJ, et al. Malignant transformation rates in oral submucous fibrosis over a 17 year period. Community Dent Oral Epidemiol 1985;13:340-1.

5. Johnson NW, Ranasinghe AW, Warnakulasuriya KAAS. Potentially malignant lesions and conditions of the mouth and oropharynx: natural history-cellular and molecular markers of risk. Eur J Cancer Prevent 1993;2:31-52.

6. Lee $\mathrm{CH}$, Ko $\mathrm{YC}$, Huang $\mathrm{HL}$, et al. The precancer risk of betel quid chewing, tobacco use and alcohol consumption in oral leukoplakia and oral submucous fibrosis in Southern Taiwan. Brit J Cancer 2003;88:366-72.

7. Siddiqui SN, Saawarn N, Nair PP, et al. Eustachian tube dysfunction in OSMF- often present seldom discovered. J Clin Exp Dent 2014;6: e369-73.

8. Yardimci G, Kutlubay Z, Engin B, et al. Precancerous lesions of oral mucosa. World J Clin Cases 2014;2:866-72.

9. Shiu MN, Chen TH. Intervention efficacy and malignant transformation to oral cancer among patients with leukoplakia. Oncol Rep 2003;10:1683-92.
10. Scheifele C, Reichart PA. Is there a natural limit of the transformation rate of oral leukoplakia? Oral Oncol 2003:39:470-5.

11. Ko YC, Huang YL, Lee $\mathrm{CH}$, et al. Betel-nut chewing, cigarette smoking and alcohol consumption related to oral cancer in Taiwan $J$ Oral Pathol Med 1995;24:450-3.

12. Wen CP, Tsai SP, Cheng TY, et al. Uncovering the relation between betel quid chewing and cigarette smoking in Taiwan. Tob Control 2005; 14:i16-22.

13. Arora D, Marya CM, Menon I, et al. Cross sectional survey on association between alcohol, betel-nut, cigarette consumption and health promoting behavior of industrial workers in Ghaziabad. Asian Pac J Cancer Prev 2015;16:139-44.

14. Lee KW, Kuo WR, Tsai SM, et al. Different impact from betel quid, alcohol and cigarette: risk factors for pharyngeal and laryngeal cancer. Int J Cancer 2005;117:831-6.

15. Prasad S, Anand R, Dhingra C. Betel nut chewing behavior and its association with oral mucosal lesions and conditions in Ghaziabad India. Oral Health Prev Dent 2014;12:241-8.

16. Yen AM, Chen SL, Chiu SY, et al. Association between metabolic syndrome and oral pre-malignancy: a community-and population-based study. Oral Oncol 2011;47:625-30.

17. Stocks T, Bjørge T, Ulmer $\mathrm{H}$, et al. Metabolic risk score and cancer risk: pooled analysis of seven cohorts. Int J Epidemiol 2015;44:1353-63.

18. Esposito K, Chiodini P, Colao A, et al. Metabolic syndrome and risk of cancer: a systematic review and meta-analysis. Diabetes Care 2012;35:2402-11.

19. Santi I, Kroll LE, Dietz A, et al. Occupation and educational inequalities in laryngeal cancer: the use of a job index. BMC Public Health 2013;13:1080.

20. Guo SE, Huang TJ, Huang JC, et al. Alcohol, betel-nut and cigarette consumption are negatively associated with health promoting behaviors in Taiwan: a cross sectional study. BMC Public Health 2013;13:257.

21. Yang YH, Lien YC, Ho PS, et al. The effects of chewing areca/betel quid with and without cigarette smoking on oral submucosa fibrosis and oral mucosal lesions. Oral Dis 2005;11:88-94.

22. Sinor PN, Gupta PC, Murti PR, et al. A case-control study of ora submucous fibrosis with special reference to the etiologic role of areca nut. J Oral Pathol Med 1990;19:94-8.

23. Tsai WC, Wu MT, Wang GJ, et al. Chewing areca nut increases the risk of coronary artery disease in Taiwanese men: a case-control study. BMC Public Health 2012;12:162.

24. World Health Organization. Calibration of examiners for oral health epidemiological surveys. Geneva: ORH/EPID, 1997.

25. Chung $\mathrm{CH}$, Yang YH, Wang TY, et al. Oral precancerous disorders associated with areca quid chewing, smoking, and alcohol drinking in southern Taiwan. $J$ Oral Pathol Med 2005:34:460-6.

26. Health Promotion Administration, Ministry of Health and Welfare. Metabolic syndrome. http://www.hpa.gov.tw/BHPNet/Web/ HealthTopic/TopicArticle.aspx? $\mathrm{No}=200712250123 \&$ parentid $=$ 200712250023 (accessed 22 Feb 2015).

27. Karmali R, Dalovisio A, Borgia JA, et al. All in the family: clueing into the link between metabolic syndrome and hematologic malignancies. Blood Rev 2015;29:71-80.

28. Lee SY, Tsai TI, Tsai YW, et al. Health literacy, health status, and healthcare utilization of Taiwanese adults: results from a national survey. BMC Public Health 2010;10:614. 